\title{
Endobronchial metastasis of osteosarcoma presenting as position-dependent and variable airway obstruction of the trachea
}

Endobronchial metastasis secondary to osteosarcoma is distinctly rare. ${ }^{12}$ We report a young man with metastatic osteosarcoma who presented with position-dependent dyspnoea. A CT scan of the chest showed a soft tissue mass that originated from the left lung, causing significant volume loss of the left lung, extended through the left main bronchus and projected to the trachea (fig 1A,B). Bronchoscopy showed a tongue-like lesion that was noted to flip back and forth from the right main bronchus on inspiration to the main trachea on expiration (fig 1C,D and video). The patient underwent left-sided pneumonectomy and the pathology confirmed the diagnosis of metastatic osteosarcoma (fig 2).

\section{VIDEO}

The video was obtained during bronchoscopy as the patient was in the supine position. It demonstrates the variable position of the tongue-like lesion flipping back and forth from the right main bronchus on inspiration to the main trachea on expiration.

\section{O Mazboudi, ${ }^{1}$ M R Abboud, ${ }^{2}$ S Muwakkit, ${ }^{2}$ S Akel, ${ }^{3}$ A Husari ${ }^{1}$ \\ ${ }^{1}$ Division of Pulmonary and Critical Care Medicine, Department of Internal Medicine, American University of Beirut - Medical Center, Beirut, Lebanon; ${ }^{2}$ Pediatric Hematology-Oncology, Department of Pediatrics, American University of Beirut - Medical Center, Beirut, Lebanon; ${ }^{3}$ Pediatric Surgery, Department of Surgery, American University of Beirut - Medical Center, Beirut, Lebanon}

Correspondence to: Dr A Husari, Department of Internal Medicine, Faculty of Medicine, American University of Beirut, P 0 Box 11-236, Riad El Solh Beirut 1107 2020 Lebanon; ah51@aub.edu.lb

Figure 1 (A) CT scan of the chest showing an endobronchial mass originating from the left main lung and projecting into the carina. The arrow shows the mass in the coronal section. (B) Arrow pointing to the mass in the sagittal section. (C) Bronchoscopic image showing a muscular tongue-like lesion located in the trachea on expiration. (D) The tongue-like lesion is withdrawn into the right main bronchus on inspiration.

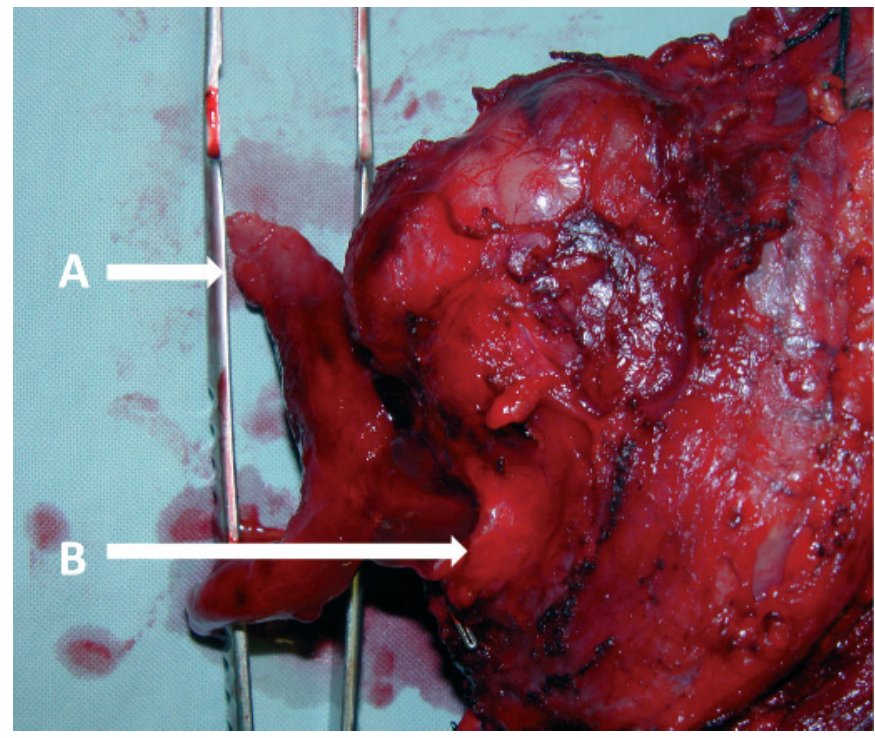

Figure 2 Gross pathology after left pneumonectomy showing the tonguelike lesion seen in fig 1 (A). The arrow (B) is pointing to the left main bronchus.

Funding:

Competing interests:

Provenance and peer review: Not commissioned; externally peer reviewed. Accepted 30 March 2009

Thorax 2010;65:92. doi:10.1136/thx.2008.112086

\section{REFERENCES}

1. Picci P. Osteosarcoma (osteogenic sarcoma). Orphanet J Rare Dis 2007;2:6.

2. Mogulkoc N, Goker E, Atasever A, et al. Endobronchial metastasis from osteosarcoma of bone: treatment with intraluminal radiotherapy. Chest 1999;116:1811-4.
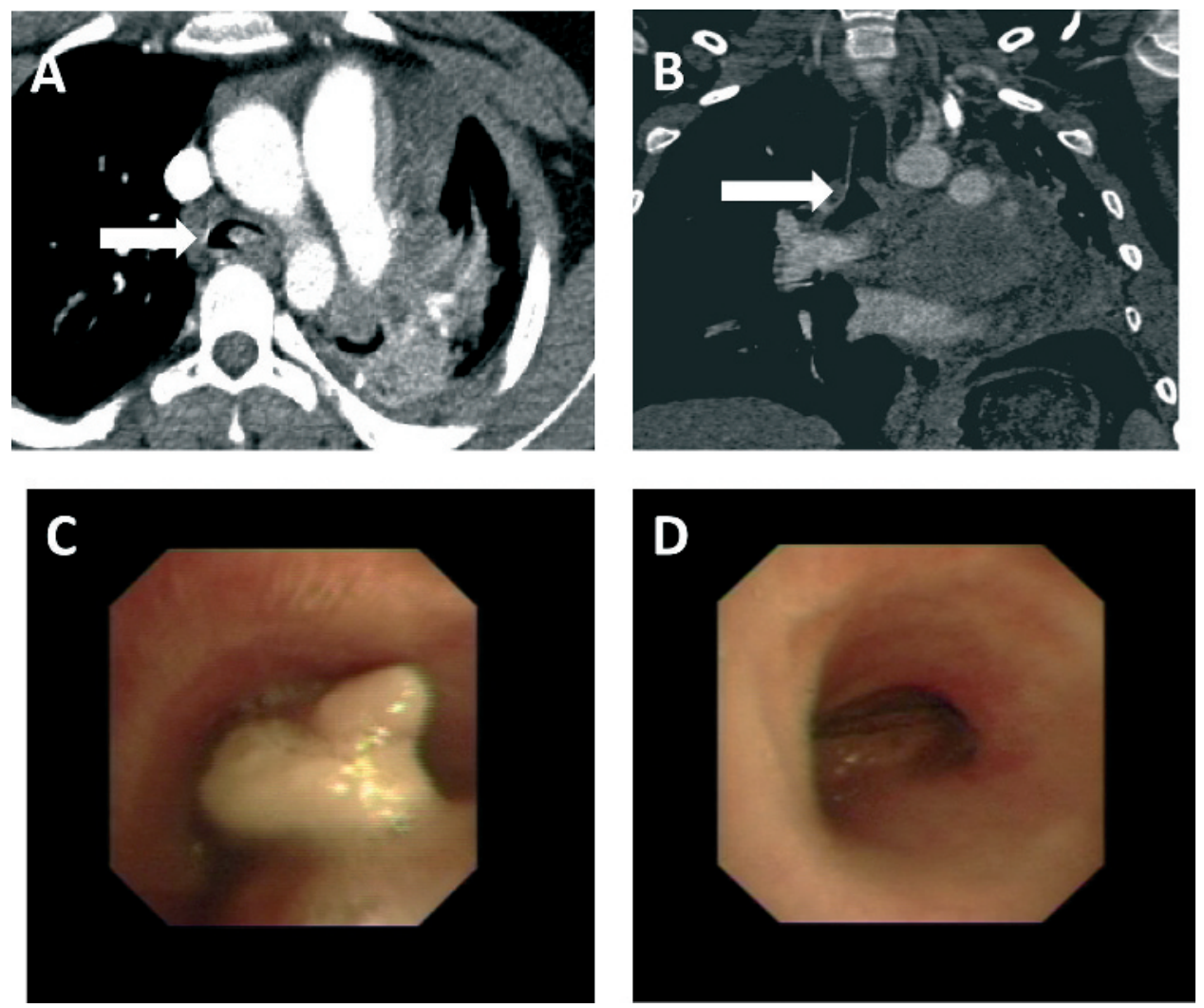